\title{
HOW TO DEFINE SINGULAR SOLUTIONS
}

\author{
By Shyujchi IzUmiya AND JiAnMing Yu
}

\begin{abstract}
We shall give a rigorous definition of singular solutions of ordinary differential equations of the form $F(x, y, d y / d x)=0$. Our main result clarifies the geometric meaning of such a definition. All arguments are elementary.
\end{abstract}

\section{Introduction.}

How to define singular solution? We shall consider this philosophical and stimulative question. However, we stick to ordinary differential equations of the form $F(x, y, d y / d x)=0$. Even for such elementary differential equations, the above question is still significant.

In classical treatises of equations (Carathéodory [2], Courant-Hilbert [3], Forsyth [4], [5], Ince [2], Petrovski [9]) the discussions of equations with singular solutions are informal. In these, a "general solution" of the differential equation $F(x, y, d y / d x)=0$ is defined to be an one-parameter family of solutions and a "singular solution" is a solution which is not contained in the "general solution". However, this definition of singular solutions is very confused as the following example shows :

Example 0.1. Consider the equation $y=2 p \cdot x-p^{2}$, where $p=d y / d x$. In [7] the "general solution" is given by

$$
\left\{\begin{array}{l}
x=\frac{c}{p^{2}}+\frac{2}{3} p \\
y=2 p \cdot x-p^{2},
\end{array}\right.
$$

where $c$ is a parameter. It is clear that $y=0$ is also a solution, but it is not contained in the "general solution". Then $y=0$ must be the "singular solution".

On the other hand, we have a two parameter family of solutions:

$$
\gamma\left({ }_{\left(c_{1}, c_{2}\right)}(t)=\left(\frac{2}{3} c_{1} e^{-t}+c_{2} e^{2 t}, \frac{1}{2} c_{1}^{2} e^{-2 t}+2 c_{1} c_{2} e^{t}, c_{1} e^{-t}\right) .\right.
$$

If we fix $p(0)=c_{1} \neq 0$ and put $c=c_{2} c_{1}^{2}$, then we have $x=(2 / 3) p+\left(c / p^{2}\right)$ and

Received November 2, 1992. 
$y=2 p \cdot x-p^{2}$. But, if we fix $p(0)=c_{1}=0$, then we have $y=0$. Moreover, if we consider this family of solutions around a point $\left(x_{0}, 0,0\right), x_{0} \neq 0$, then we have the relation $x(0)=(2 / 3) c_{1}+c_{2}=x_{0}$, so that, we have an one-parameter family of "solutions" around $\left(x_{0}, 0,0\right)$ :

$$
\gamma_{c_{1}}(t)=\left(\frac{2}{3} c_{1} e^{-t}+\left(x_{0}-\frac{2}{3} c_{1}\right) e^{2 t}, \frac{1}{3} c_{1}^{2} e^{-2 t}+2 c_{1}\left(x_{0}-\frac{2}{3} c_{1}\right) e^{t}, c_{1} e^{-t}\right) .
$$

Of course, $y=0$ is contained in this family of solutions.

In this note we intend to give a rigorous definition of singular solutions of first order ordinary differential equations of the form $F(x, y, d y / d x)=0$. In [6] M. and T. Fukuda tried to give a rigorous definition of singular solutions of higher order ordinary differential equations. Their definition is nearly correct, however the definition of singular solutions is strongly depend on the definition of general solutions. The vagueness of the definition of singular solutions is caused by that of general solutions, so that we should begin to define "general solutions" in the correct way. The main results of this note can be generalized to the case of partial differential equations ([8], [10]). In these articles, we need some techniques of contact geometry. Here, we shall only use a purely elementary method; most of the arguments are contained in the course of advanced calculus in the university.

All functions and mappings considered here are differentiable of class $C^{\infty}$, unless stated otherwise.

\section{Basic notions.}

We consider a first order ordinary differential equation of the form $F(x, y, d y / d x)=0$. If we put $p=d y / d x$, we may consider $F$ as a function of $(x, y, p)$ and assume that $F$ is a smooth function defined on an open subset $U$ in $\boldsymbol{R}^{3}$ such that $\operatorname{grad} F \neq 0$ at any point $(x, y, p) \in U$. Then $S=F^{-1}(0)$ is a smooth surface in $U$.

We now define the notion of solutions. A smooth solution of $F=0$ is a smooth function $y=f(x)$ defined on an interval $(a b) \subset \boldsymbol{R}$ such that $F(x, f(x)$, $\left.f^{\prime}(x)\right)=0$. This is the classical notion of solutions of the equation $F=0$. The following is the geometric generalization of the notion of solution due to Lie. A geometric solution of $F=0$ is a smooth regular curve $\gamma:(a b) \rightarrow F^{-1}(0)$ such that $y^{\prime}(t)=p(t) x^{\prime}(t)$ and $\gamma(t)=(x(t), y(t), p(t))$ in the canonical coordinates system of $\boldsymbol{R}^{3}$. Here, we say that $\gamma$ is regular if $\gamma^{\prime}(t)=\left(x^{\prime}(t), y^{\prime}(t), p^{\prime}(t)\right) \neq(0,0,0)$ at any $t \in(a b)$. In the terminology of contact geometry, the above curve is called a Legendrian curve (see [1]). Then we can prove the following simple lemma (just an exercise for students).

LeMMA 1.1. Let $\gamma:(a b) \rightarrow F^{-1}(0)$ be a geometrac solution. Suppose that $x^{\prime}(t)$ $\neq 0$ at any $t \in(a b)$. Then there exist real numbers $c, d$, diffeomorphism $\phi:\left(\begin{array}{ll}c & d\end{array}\right)$ 
$\rightarrow\left(\begin{array}{ll}a & b\end{array}\right)$ and a smooth function $f$ defined on $\left(\begin{array}{cl}c & d\end{array}\right)$ such that $\gamma \circ \phi(x)=\left(x, f(x), f^{\prime}(x)\right)$.

According to the above property, we may define the notion of singular point of solutions. We say that $t_{0}$ is a geometric singular point of the solution $\gamma$ if $x^{\prime}\left(t_{0}\right)=0$. Thus $\gamma$ is multivalued around the geometric singular point. It is clear that $t_{0}$ is a geometric singular point of $\gamma$ if and only if $\left(x^{\prime}\left(t_{0}\right), y^{\prime}\left(t_{0}\right)\right)=$ $(0,0)$.

On the other hand, there exists a notion of the Legendrian transformation by which a dual relationship can be set up between one equation and another. We adopt another coordinate system $(X, Y, P)$ of $\boldsymbol{R}^{3}$ by $X=p, Y=x \cdot p-y$, $P=x$. We refer to the diffeomorphism $* L: \boldsymbol{R}^{3} \rightarrow \boldsymbol{R}^{3}$ defined by $* L(x, y, p)=$ $(p, x \cdot p-y, x)$ as a Legendre transformation. By the definition, we have $* L^{-1}(X, Y, P)=(P, X \cdot P-Y, X)$. If we apply the Legendre transformation to our equation, we obtain a new equation

$$
F^{*}(X, Y, P)=F_{\circ}(* L)^{-1}(X, Y, P)=F(P, X \cdot P-Y, X)=0
$$

in the new coordinate system $(X, Y, P)$.

If we calculate partial derivatives at the point $\left(X_{0}, Y_{0}, P_{0}\right)$ corresponding to $\left(x_{0}, y_{0}, p_{0}\right)$, we can show the following:

$$
\begin{aligned}
& F_{P}^{*}\left(X_{0}, Y_{0}, P_{0}\right)=\left(F_{x}+p \cdot F_{y}\right)\left(x_{0}, y_{0}, p_{0}\right) \\
& F_{Y}^{*}\left(X_{0}, Y_{0}, P_{0}\right)=-F_{y}\left(x_{0}, y_{0}, p_{0}\right) \\
& F_{X}^{*}\left(X_{0}, Y_{0}, P_{0}\right)=\left(F_{p}+x \cdot F_{y}\right)\left(x_{0}, y_{0}, p_{0}\right) .
\end{aligned}
$$

The following lemma is quite simple but important in the later section.

LEMMA 1.2. (1) Let $\gamma:(a b) \rightarrow F^{-1}(0)$ be a geometric solution of $F=0$. Then $* L \circ \gamma:(a b) \rightarrow F^{*-1}(0)$ is a geometric solution of $F^{*}=0$.

(2) If $t_{0}$ is a geometric singular point of $\gamma$, then $t_{0}$ is a geometric nonsingular point of $* L \circ \gamma$.

If the equation $F=0$ satisfies $F_{p} \neq 0$ at $\left(x_{0}, y_{0}, p_{0}\right)$, then we can locally rewrite this equation in the form $p=f(x, y)$, where $f$ is a smooth function by the implicit function theorem. This form is far more convenient than the original one, because there exists the classical existence theorem of solutions. By the above argument, if $F=0$ satisfies $F_{x}+p \cdot F_{y} \neq 0$ at $\left(x_{0}, y_{0}, p_{0}\right)$, then the Legendre transformed equation $F^{*}=0$ of $F=0$ satisfies $F_{P}^{*} \neq 0$ so that we get a solution of $F^{*}=0$. Since $F=F^{*} \circ(* L)$, then we have a geometric solution of $F=0$ by Lemma 1.2. Then the point $\left(x_{0}, y_{0}, p_{0}\right)$ at which $F=F_{p}=0$ or $F=F_{p}=$ $F_{x}+p \cdot F_{y}=0$ are satisfied has special meanings. We call $\left(x_{0}, y_{0}, p_{0}\right) a \pi$-singular point of $F=0$ if $F=F_{p}=0$ at $\left(x_{0}, y_{0}, p_{0}\right)$ and a contact singular point of $F=0$ if $F=F_{p}=F_{x}+p \cdot F_{y}=0$. We denote $\Sigma_{\pi}(F)$ as the set of $\pi$-singular points, $\Sigma_{c}(F)$ as the set of contact singular points and $D_{F}=\pi\left(\Sigma_{\pi}(F)\right)$ as the discriminant set 
of $F=0$, where $\pi(x, y, p)=(x, y)$. The following lemma shows the importance of the contact singular set $\Sigma_{c}(F)$.

LEMMA 1.3. Let $\gamma:(a b) \rightarrow F^{-1}(0)$ be a smooth regular curve. If Image $\gamma \subset$ $\Sigma_{c}(F)$, then $\gamma$ is a geometric solution.

Proof. Denote $\gamma(t)=(x(t), y(t), p(t))$, then we have

$$
F(x(t), y(t), p(t))=F_{p}(x(t), y(t), p(t))=\left(F_{x}+p \cdot F_{y}\right)(x(t), y(t), p(t))=0 .
$$

If $F_{y}=0$ at $\gamma(t)$, then $F_{p}=F_{x}=0$ at $\gamma(t)$ by the above equality. This contradicts to the assumption that $\operatorname{grad} F \neq 0$, so that we have $F_{y}(x(t), y(t), p(t)) \neq 0$. Calculating the derivative of $F(x(t), y(t), p(t))=0$ with respect to $t$, we have

$$
F_{x}(x(t), y(t), p(t)) \cdot x^{\prime}(t)+F_{y}(x(t), y(t), p(t)) \cdot y^{\prime}(t)=0 .
$$

It follows that

$$
y^{\prime}(t)=-\frac{F_{x}(x(t), y(t), p(t))}{F_{y}(x(t), y(t), p(t))} \cdot x^{\prime}(t)
$$

By the relation (*), we have

$$
p(t)=-\frac{F_{x}(x(t), y(t), p(t))}{F_{y}(x(t), y(t), p(t))},
$$

so that we have the relation $y^{\prime}(t)=p(t) \cdot x^{\prime}(t)$.

\section{Results.}

In order to avoid the confusion as in Example 0.1, we now introduce the following notion. Let $\Gamma:(a b) \times(\alpha \beta) \rightarrow F^{-1}(0)$ be an one-parameter family of geometric solutions of $F=0$. We say that $\Gamma$ is a complete solution if

$$
\operatorname{rank}\left(\begin{array}{lll}
x_{t} & y_{t} & p_{t} \\
x_{c} & y_{c} & p_{c}
\end{array}\right)=2
$$

at any point $(t, c) \in(a b) \times(\alpha \beta)$, where $\Gamma(t, c)=(x(t, c), y(t, c), p(t, c))$ and $c$ is a parameter. If the image of $\Gamma$ contains a point $\left(x_{0}, y_{0}, p_{0}\right) \in F^{-1}(0)$, we call it a complete solution around $\left(x_{0}, y_{0}, p_{0}\right)$. In some classical textbooks (cf. [9]), the above term is used in a different sense. However, we adopt the above definition according to the terminology in the theory of first-order partial differential equations ([2], [3]). We say that an equation $F=0$ is completely integrable around $\left(x_{0}, y_{0}, p_{0}\right)$ if there exists a complete solution of $F=0$ around $\left(x_{0}, y_{0}, p_{0}\right)$. The equation in Example 0.1 is not completely integrable around the origin. We now state a basic property of complete integrable equations.

Proposition 2.1. Suppose that $F=0$ is completely integrable around $\left(x_{0}, y_{0}, p_{0}\right)$, 
then $\left(x_{0}, y_{0}, p_{0}\right) \notin \Sigma_{c}(F)$ or $\Sigma_{c}(F)$ is an 1-dimensional submanifold around $\left(x_{0}, y_{0}, p_{0}\right)$.

Proof. Let $\Gamma:(a b) \times(\alpha \beta) \rightarrow F^{-1}(0)$ be a complete solution of $F=0$ around $\left(x_{0}, y_{0}, p_{0}\right)$. Suppose that $\left(x_{0}, y_{0}, p_{0}\right) \in \Sigma_{c}(F)$, then $F_{y} \neq 0$ at $\left(x_{0}, y_{0}, p_{0}\right)$ by the same reason as that in the proof of Lemma 1.3.

We now differentiate the both sides of the equality $F(x(t, c), y(t, c), p(t, c))$ $=0$ with respect to $c$, where $\Gamma(t, c)=(x(t, c), y(t, c), p(t, c))$ and $c$ is a parameter. Then we have $F_{x} \cdot x_{c}+F_{y} \cdot y_{c}+F_{p} \cdot p_{c}=0$. It follows from the definition that $\Gamma(t, c) \in \Sigma_{c}(F)$ if and only if $F_{y} \cdot\left(y_{c}-p \cdot x_{c}\right)=0$. Since $F_{y} \neq 0$ at $\Gamma(t, c)$, the above equality is equivalent to $y_{c}(t, c)-p(t, c) \cdot x_{c}(t, c)=0$. Thus we have

$$
\Sigma_{c}(F)=\left\{(x(t, c), y(t, c), p(t, c)) \mid y_{c}(t, c)-p(t, c) \cdot x_{c}(t, c)=0\right\} .
$$

Since $\Gamma$ is a complete solution, we have $y_{t}(t, c)=p(t, c) \cdot x_{t}(t, c)$, so that $y_{t c}(t, c)=p_{c}(t, c) \cdot x_{t}(t, c)+p(t, c) \cdot x_{t c}(t, c)$.

On the other hand, $\Gamma^{-1}\left(\Sigma_{c}(F)\right)$ is defined by the equation $f(t, c)=y_{c}(t, c)-$ $p(t, c) . \quad x_{c}(t, c)=0$. If we have $f_{t}(t, c)=y_{c t}(t, c)-p_{t}(t, c) \cdot x_{c}(t, c)+p(t, c) \cdot x_{c t}(t, c)$ $=0$, then $p_{t}(t, c) \cdot x_{c}(t, c)-p_{c}(t, c) \cdot x_{t}(t, c)=0$. This contradicts to the fact that

$$
\operatorname{rank}\left(\begin{array}{lll}
x_{t} & y_{t} & p_{t} \\
x_{c} & y_{c} & p_{c}
\end{array}\right)=2 .
$$

Hence, $f_{t}(t, c) \neq 0$ at $(t, c) \in \Gamma^{-1}\left(\Sigma_{c}(F)\right)$. This completes the proof.

If $F_{p} \neq 0$ at $\left(x_{0}, y_{0}, p_{0}\right)$, it is already mentioned in $\S 1$ and that there exists a unique solution of $F=0$ by the classical existence theorem and this gives a complete solution around $\left(x_{0}, y_{0}, p_{0}\right)$. We can also have a complete solution of $F=0$ around $\left(x_{0}, y_{0}, p_{0}\right) \notin \Sigma_{c}(F)$ by the argument about the Legendre transformation in $\S 1$. In fact, we can prove local uniqueness of complete solutions around any points, however we do not need this fact. Then we omit the proof.

We now give a rigorous definition of singular solutions. Let $\gamma:(a b) \rightarrow$ $F^{-1}(0)$ be a geometric solution such that $\gamma\left(t_{0}\right)=\left(x_{0}, y_{0}, p_{0}\right)$. We say that $\gamma$ is $a$ singular solution of $F=0$ around $\left(x_{0}, y_{0}, p_{0}\right)$, if it is never contained in any complete solution around $\left(x_{0}, y_{0}, p_{0}\right)$. We also say that $\gamma$ is a singular solution of $F=0$ in the strict sense around $\left(x_{0}, y_{0}, p_{0}\right)$ for any open subinterval $(c d) \subset$ $\left(\begin{array}{ll}a & b\end{array}\right), \gamma \mid\left(\begin{array}{ll}c & d\end{array}\right)$ is never contained in any complete solutions of $F=0$.

Returning to the equation $y=2 p \cdot x-p^{2}$ in Example 0.1 , we can easily show that $\Sigma_{c}(F)=\{(0,0,0)\}$. By Proposition 2.1, this equation is not completely integrable around $(0,0,0)$. It follows that the solution $y=0$ is the singular solution around $(0,0,0)$. However, it is not a singular solution in the strict sense. The following theorem describes the relation between singular solutions and singular solutions in the strict sense.

THEOREM 2.2. For an equation $F=0$ and $a$ geometric solution $\gamma:(a b) \rightarrow$ 
$F^{-1}(0)$ such that $\gamma\left(t_{0}\right)=\left(x_{0}, y_{0}, p_{0}\right)$, the following are equivalent.

(0) $F=0$ is completely integrable around $\left(x_{0}, y_{0}, p_{0}\right)$ and $\gamma$ is a singular solution of $F=0$ around $\left(x_{0}, y_{0}, p_{0}\right)$.

(1) $\gamma$ is a singular solution of $F=0$ in the strict sense.

(2) There exists a complete solution around any point of $\gamma\left(\left(\begin{array}{ll}a & b\end{array}\right)\right)$ such that each member is transverse to $\gamma$.

(3) Image $\gamma=\Sigma_{c}(F)$.

Proof. (3) $\Rightarrow(2)$. By the same reason as that of the proof of Lemma 1.3, we have $F_{y} \neq 0$ at $\gamma(0)$. By the implicit function theorem, the equation $F=0$ can be rewritten as $y=h(x, p)$ around $\gamma\left(t_{0}\right)$, so that, we have

$$
\Sigma_{c}(y-h(x, p))=\left\{(x, h(x, p), p) \mid h_{p}(x, p)=h_{x}(x, p)-p=0\right\} .
$$

Then we may distinguish two cases:

(a) $h_{x p}(x, p)-1=0$,

(b) $h_{x p}(x, p)-1 \neq 0$.

Case (a). Since $h_{x p}=h_{p x}=1 \neq 0$ at $\left(x\left(t_{0}\right), p\left(t_{0}\right)\right)$, the set

$$
\Sigma_{\pi}(y-h(x, p))=\left\{(x, h(x, p), p) \mid h_{p}=0\right\}
$$

is a smooth curve. $\gamma$ is also a smooth curve, then we have $\gamma((a b))=\Sigma_{c}(y-h(x, p))$ $=\Sigma_{\pi}(y-h(x, p))$. It follows that there exists a smooth function $\mu$ around $\left(x\left(t_{0}\right), p\left(t_{0}\right)\right)$ such that $\mu$ does not vanish on such a neighbourhood and $h_{x}-p$ $=\mu \cdot h_{p}$.

We now consider a vector field on the $(x, p)$-plane defined by

$$
V=\frac{\partial}{\partial x}-\mu \cdot \frac{\partial}{\partial p} .
$$

By the same reason as in Example 0.1, the flows of the vector field $V$ gives a complete solution of $y-h(x, p)=0$ around $\gamma\left(t_{0}\right)$.

On the other hand, by the previous arguments, the curve $(x(t), p(t))$ is given by the equation $h_{p}=0$ near $\left(x\left(t_{0}\right), p\left(t_{0}\right)\right)$. If we calculate the canonical inner product of $\operatorname{grad} h_{p}$ and $V$, then we have

$$
\left\langle\operatorname{grad} h_{p}, V\right\rangle=1-\mu \cdot h_{p p} .
$$

Differentiate the equation $h_{x}-p=\mu \cdot h_{p}$ with respect to $p$, then we have $\mu \cdot h_{p p}$ $=0$ at $\left(x\left(t_{0}\right), p\left(t_{0}\right)\right)$. It follows that $\left\langle\operatorname{grad} h_{p}, V\right\rangle \neq 0$ at $\left(x\left(t_{0}\right), p\left(t_{0}\right)\right)$, so that $V$ does not tangent to $\gamma$. This means that each member of the complete solution is transverse to $\gamma$ around $\gamma\left(t_{0}\right)$.

Case (b). In this case the set $\left\{(x, h(x, p), p) \mid h_{x}(x, p)-p=0\right\}$ is a smooth curve near $\left(x\left(t_{0}\right), y\left(t_{0}\right), p\left(t_{0}\right)\right)$. By the same reason as that of the case (a), there exists a smooth function $\lambda$ around $\left(x\left(t_{0}\right), p\left(t_{0}\right)\right)$ which never vanish and satisfies $h_{p}=\lambda \cdot\left(h_{x}-p\right)$. We adopt a vector field $V=\lambda \cdot \partial / \partial x-\partial / \partial p$ on $(x, p)$-plane, so that the flow of $V$ gives a required complete solution. 
$(2) \Rightarrow(1)$. If $\gamma$ is not a singular solution in the strict sense, then there exist an intervals $\left(a^{\prime} b^{\prime}\right) \subset(a b),\left(\alpha^{\prime} \beta^{\prime}\right) \subset(\alpha \beta)$ and a complete solution $\Gamma:\left(\begin{array}{ll}a^{\prime} & b^{\prime}\end{array}\right) \times$ $\left(\alpha^{\prime} \beta^{\prime}\right) \rightarrow F^{-1}(0)$ such that $\Gamma_{c_{0}}=\gamma$ for some $c_{0} \in\left(\alpha^{\prime} \beta^{\prime}\right)$. By Proposition 2.1 and the previous proof, there exists $t_{0} \in\left(a^{\prime} b^{\prime}\right)$ such that $\Gamma\left(t_{0}, c_{0}\right) \notin \Sigma_{c}(F)$. By the local uniqueness of the complete solution outside of $\Sigma_{c}(F)$, the complete solution $\Gamma$ is equal to the original complete solution on some open neighbourhood of $\Gamma\left(t_{0}, c_{0}\right)$ which is given by the condition (2). However, each member of this complete solution must be transverse to $\gamma=\Gamma_{c_{0}}$. This gives a contradiction.

$(1) \Rightarrow(3)$. If Image $\gamma \not \subset \Sigma_{c}(F)$, then there exists $t_{0} \in(a b)$ such that $\gamma\left(t_{0}\right) \notin \Sigma_{c}(F)$. It follows that $F_{p} \neq 0$ or $F_{x}+p \cdot F_{y} \neq 0$ at $\gamma\left(t_{0}\right)$. In both cases, there exists a unique complete solution of $F=0$ which contains $\gamma$ around $\gamma\left(t_{0}\right)$. This contradicts to the definition of singular solutions in the strict sense.

Here, we only proved that Image $\gamma \subset \Sigma_{c}(F)$ instead of the condition (3), however, by the previous proof and Proposition 2.1, we can assert that Image $\gamma=$ $\Sigma_{c}(F)$.

$(0) \Rightarrow(3)$. Let $\Gamma:(a b) \times(\alpha \beta) \rightarrow F^{-1}(0)$ be a complete solution of $F=0$ around $\left(x_{0}, y_{0}, p_{0}\right) \in \Sigma_{c}(F)$. If Image $\gamma \neq \Sigma_{c}(F)$, then there exists $t_{0} \in(a b)$ such that $\gamma\left(t_{0}\right) \notin \Sigma_{c}(F)$. It follows that $F_{p} \neq 0$ or $F_{x}+p \cdot F_{y} \neq 0$ at $\gamma\left(t_{0}\right)$. In both cases, there exists a unique complete solution of $F=0$ which contains $\gamma$ around $\gamma\left(t_{0}\right)$. This complete solution is equal to $\Gamma$ around $\gamma\left(t_{0}\right)$. Hence, Image $\gamma=$ Image $\Gamma_{c_{0}}$ around $t_{0}$, where $\Gamma_{c_{0}}(t)=\Gamma\left(t, c_{0}\right)$. This solution eventually reaches $\left(x_{0}, y_{0}, p_{0}\right)$, so that it is contained in $\Gamma$ around $\left(x_{0}, y_{0}, p_{0}\right)$. This contradicts to the definition of singular solutions.

It is clear that $(1)$ and $(2) \Rightarrow(0)$. This completes the proof.

The following is a classical example of an equation with singular solution.

Example 2.3. The Clairaut equation: $y=x \cdot p+f(p)$.

The singular solution is $\Sigma_{c}(F)=\Sigma_{\pi}(F)$ and the discriminant set $D_{F}$ is the envelope of the complete solution $y=x \cdot c+f(c)$.

In classical treatises, it has been considered that $\Sigma_{\pi}(F)$ was a strong candidate for the singular solution as the Clairaut equation shows. However, Theorem 2.2 asserts that $\Sigma_{c}(F)$ is the singular solution in the strict sense.

Example 2.4. $y=x \cdot p^{2}+p^{3}$.

We can calculate that $\Sigma_{\pi}(F)=\left\{(x, y, p) \mid y=x \cdot p^{2}+p^{3}\right.$ and $\left.p \cdot(2 x+3 p)=0\right\}$ and $\Sigma_{c}(F)=\{(x, y, p) \mid y=p=0\}$. By Theorem 2.2, this equation is completely integrable around $(0,0,0)$ and the singular solution is given by $y=p=0$.

Acknowledgment. A part of this work was done during the first author's stay at the University of Liverpool. The first author would like to thank all members of the Department of Pure Mathematics for giving him a chance to talk about these subjects at the internal colloquium. This work was completed 
during the authors' stay at Guizhou University in China. The authors are also grateful to Professor Guobin Zhang for his kind hospitality.

\section{REFERENCES}

[1] V.I. ARNol'D, Contact geometry and wave propagation, Monographie de L'Enseignement Mathematique 34 (1989).

[2] C. Carathéodory, Calculus of Variations and Partial Differential Equations of First Order, Part I, Partial Differential Equations of the First Order, HoldenDay, 1965.

[3] R. Courant and D. Hilbert, Methods of mathematical physics I, II, Wiley, New York, 1962.

[4] A.R. Forsyth, A Treatise on differential equations, Macmillan and Co, 1885.

[5] A.R. Forsyth, Theory of differential equations, Part III partial differential equations. Cambridge Univ. Press, London, 1906.

[6] M. FukUda AND T. FukUda, Singular solutions of ordinary differential equations, The Yokohama Math. Jour. 15 (1977), 41-58.

[7] E. L. INCE, Ordinary differential equations, Dover, 1926.

[8] S. IzumiYa, Singular solutions of first order differential equations, to appear in Bull. London Math. Soc..

[9] I.G. Petrovski, Ordinary differential equations, Prentice-Hall, 1996.

[10] J. YU, On singular solutions of completely integrable partial differential equations of first order, in preparation.

Department of Mathematics, Faculty of Science, HOKKAIDO UNIVERSITY, SAPPORO 060, JAPAN

Institute of Mathematics, Academia Sinica,

BEIJING 100080, P.R. China 